\title{
DEVELOPMENT OF COUETTE-TAYLOR CRYSTALLIZER IN COOLING CRYSTALLIZATION: PROMOTION OF SIZE DISTRIBUTION OF L-LYSINE
}

\author{
Dang Truong Giang, Khuu Chau Quang, Phan Thanh Thao, \\ Trinh Thi Thanh Huyen", Nguyen Anh Tuan* \\ Institute of Chemical Technology, VAST, 1 Mac Dinh Chi, District 1, HCMC \\ *Email: thanhhuyenkhtn@yahoo.com,nhtnat@yahoo.com
}

Received: 23 December 2015; Accepted for publication: 11 April 2016

\begin{abstract}
The Couette-Taylor (CT) crystallizer was developed to promote the size distribution of LLysine crystal product in cooling crystallization. When using the CT crystallizer, the size distribution of crystal product was much narrower than that of the conventional Stirred tank (ST) crystallizer. Here, the coefficient size distribution (n) in CT crystallizer was quite large as 3.43, while it was only 2.17 in ST crystallizer at the same $360 \mathrm{rpm}$ of agitation or rotation speed. This result indicated that the CT crystallizer was much more effective than the ST crystallizer in terms of the size distribution of L-lysine crystal products in cooling crystallization. The advantage of CT crystallizer over the ST crystallizer was explained in terms of the high energy dissipation of Taylor vortices flow, where it was 7.6 times higher than that of random fluid motion in conventional ST crystallizer. As such, the supersaturation profile in the CT crystallizer was much more homogeneous than that in the ST crystallizer, which resulted in promotion of size distribution L-lysine crystal product.
\end{abstract}

Keywords: crystallization, nucleation, crystal growth, agglomeration/Breakage, Couette-Taylor crystallizer.

\section{INTRODUCTION}

Crystallization is a very important separation, purification and particle synthesis process, which is certainly required in numerous industries such as foods, pharmaceuticals, chemicals and agriculture in order to obtain the high quality of crystal products including purity, size and size distribution, shape, polymorphism, etc [1]. Although crystallization has a long history, it has not been well understood yet because the mechanisms of nucleation, crystal growth, agglomeration/breakage of each material in crystallization are very distinguished and complicated.

Crystal size distribution is a significant property of crystal product since it directly impacts on the crystal behavior including dissolution rate, tableting and bioactivity, etc. Furthermore, the downstream processes such as filtration, drying and milling are strongly affected by the size 
distribution of crystal product [1]. Thus, many manufactures have paid much attention to control the size distribution of crystal product in crystallization. Generally, the size distribution of crystal product depends on many crystallization conditions including fluid hydrodynamic, supersaturation, seeding effects, dissolution of fine crystals, additive, etc. For example, Alvarez et al. [2] reported that the size distribution of flufenamic acid was promoted as increasing the mixing condition by using the static mixer in the Plug flow crystallizer. Here, the coefficient variation of size distribution was 0.9 as using the conventional Plug flow crystallizer, yet it was significantly promoted to 0.75 as using a static mixer in the Plug flow crystallizer. The effect of fluid hydrodynamic on the size distribution of barium sulfate was also reported by Baldyga et al. [3], where it was narrower as increasing the agitation speed of impeller, etc.

The CT crystallizer has a unique hydrodynamic Taylor vortices flow, which is being widely applied in many crystallization processes including batch or continuous system in order to achieve the desired properties of crystal product including purity, shape, size, size distribution, polymorphic crystals and productivity [4-6], etc. For example, according to Tuan et al. [4 - 5] when using the CT crystallizer, the phase transformation of GMP from amorphous phase to crystalline hydrate phase was significantly facilitated over 5.0 times than that in the conventional ST crystallizer, implying that the productivity of crystalline hydrate product was remarkably enhanced as using the CT crystallizer. Jung et al. [6] also indicated that the cubic shape of calcium carbonate was consistently uniform as using the CT crystallizer, while it is often a random shape of cubic, oval and needle as using the conventional ST crystallizer.

In Vietnam, even though some research have partly related with the crystallization studies, these crystallization studies have not been published on any ISI journals, meaning that it does not have good enough crystallization background and new idea. In case of Couette-Taylor crystallizer, there is no doubt to confirm that our patent Couette-Taylor (CT) crystallizer is firstly applied in Vietnam. For the L-lysine amino acid, it was chosen as the model crystal product to demonstrate the effectiveness of CT crystallizer with respect to the size distribution compared to that of the conventional ST crystallizer. According to CJ company (Korea), the size distribution of L-lysine crystal product was very broad as using the conventional ST crystallizer, implying that a new crystallization process was necessary to be developed. Thus, in present study the Couette-Taylor crystallizer was developed to promote the size distribution of L-lyine crystal product in cooling crystallization, and of course our current study was original in the

world. To evaluate the advantage of CT crystallizer over the conventional ST crystallizer, the cooling crystallization of L-lysine was conducted in both crystallizers at the same crystallization condition.

\section{EXPERIMENTAL}

The batch CT and ST crystallizer were designed by Tuan et al. [4 - 5], where the cooling jacket was installed outside of both crystallizers in order to control the temperature. During the cooling crystallization, the temperature of both crystallizers was controlled via the circulating coolant from the chiller, in which the cooling rate was always fixed as $4.0^{\circ} \mathrm{C} / \mathrm{min}$. The L-Lysine monohydrochloride crystal (> $98.5 \%$ purity) was purchased from the CJ Company (Korea), where the feed solution was prepared by dissolving the raw material in the distilled water at $50{ }^{\circ} \mathrm{C}$ with $1000(\mathrm{~g} / \mathrm{L})$ concentration. Initially, the CT and ST crystallizer were both filled with the feed solution at $50{ }^{\circ} \mathrm{C}$, and then operated as the batch mode crystallization until the suspension temperature approached to $30{ }^{\circ} \mathrm{C}$ equilibrium. By comparison, the same crystallization conditions including feed temperature, feed concentration, and rotation or 
agitation speed were carried out for both CT and ST crystallizer. The suspension samples were intermittently taken from the crystallizers and quickly filtered via the vacuum pump. The crystal products were harvested and kept in a desiccator for analyzing the size distribution. Here, the size distribution of crystal product was estimated via the Video microscope and Sieving, while the crystal structure was confirmed by X-Ray patterns (M18XHF-SRA, Japan), Thermogravimetric analysis (TGA) and Differential scanning calorimetry (DSC), respectively.

\section{RESULTS AND DISCUSSION}

\subsection{Characteristic of L-lysine crystal}

The L-lysine crystal product was the dihydrate form in both CT and ST crystallizer. Here, the crystal structure of dihydrate form was clearly distinguished with that of the anhydrous form via the characteristic peaks of XRD pattern at $13.2^{0}, 16.6^{0}, 24.6^{0}, 26.7^{0}, 31.1^{0}, 38.8^{0}$ as shown in Fig. 1. Moreover, the amount of water incorporated into the crystal lattice was confirmed as 16.5 \%wt by using Thermogravimetric analysis (TGA), corresponding to the dihydrate form, as depicted in Fig. 2.

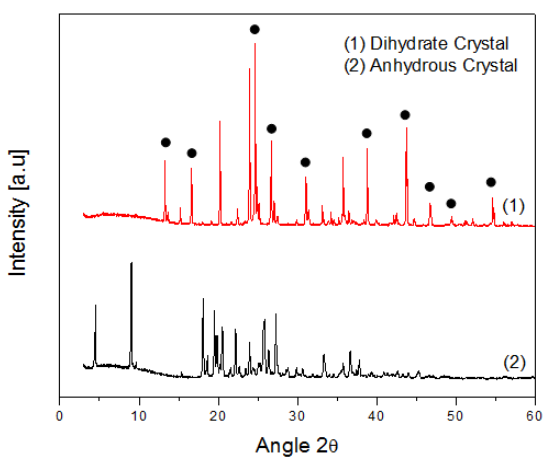

Figure 1. XRD pattern of dihydrate and anhydrous form of L-lysine crystal product.

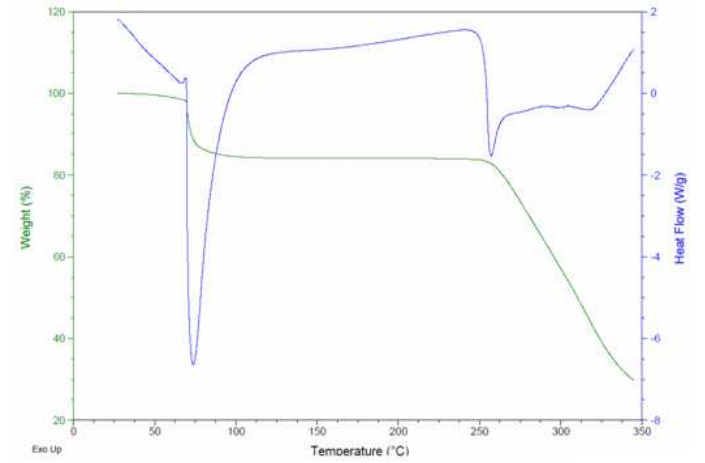

Figure 2. Thermogravimetric analysis (TGA) and Differential scanning calorimetry (DSC) of dihydrate form of L-lysine crystal product.

\subsection{Crystallization in Couette-Taylor (CT) and Stirred tank (ST) crystallizers}

The cooling crystallization of L-lysine was conducted in both CT and ST crystallizers at the same crystallization conditions. Yet, the size distribution of crystal product had a significant difference between CT and ST crystallizer, as depicted in Figs. 3 - 6. Here, the coefficient size distribution (n) was estimated via the Rosin Rammler Sperling Bennet (RRSB) method [7]. In case of conventional ST crystallizer, when the crystallization was carried out at $360 \mathrm{rpm}$, the crystal product was a mixture of small and big crystals and obviously un-uniformed, as shown in Fig. 3(a). This result revealed that the supersaturation profile in ST crystallizer was definitely non-homogeneous, leading to inducing a spontaneous nucleation and random crystal growth rate, which resulted in broad size distribution. Thus, the size distribution of crystal product was promoted if the supersaturation profile was more homogeneous. Indeed, when increasing the agitation speed from $360 \mathrm{rpm}$ to $700 \mathrm{rpm}$, the crystal size of L-lysine was more uniform, as depicted in Fig. 3(b). This result was consistent with the size distribution profiles, where the coefficient size distribution (n) increased from 2.17 to 2.45 as increasing the agitation speed from $360 \mathrm{rpm}$ to $700 \mathrm{rpm}$, as displayed in Fig. 4. As such, the size distribution of L-lysine 
crystal product was promoted as using an effective fluid hydrodynamic in cooling crystallization. In case of the CT crystallizer, the crystal size was more mono-dispersion compared to that of the conventional ST crystallizer at the same crystallization conditions, as shown in Fig. 5. As regards the size distribution profile, the coefficient size distribution (n) in CT crystallizer was quite large as varying from 3.43 to 4.35 as increasing the rotation speed from $360 \mathrm{rpm}$ to $700 \mathrm{rpm}$, while it only varied from 2.17 to 2.45 as using the conventional ST crystallizer, meaning that the size distribution of CT crystallizer was much narrowly dispersed compared to that of ST crystallizer, as depicted in Fig. 6. Here, it should be mentioned that the size distribution in CT crystallizer at $360 \mathrm{rpm}$ was even narrower than that of ST crystallizer at $700 \mathrm{rpm}$. This result indicated that the supersaturation profile generated under the Taylor vortex flow in CT crystallizer was much more homogeneous than that induced by the random fluid motion in ST crystallizer.

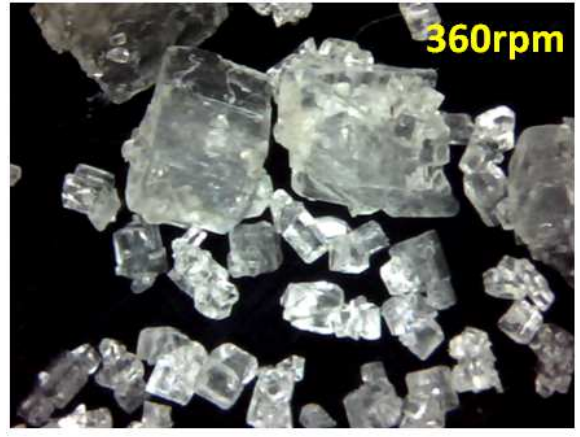

(a)

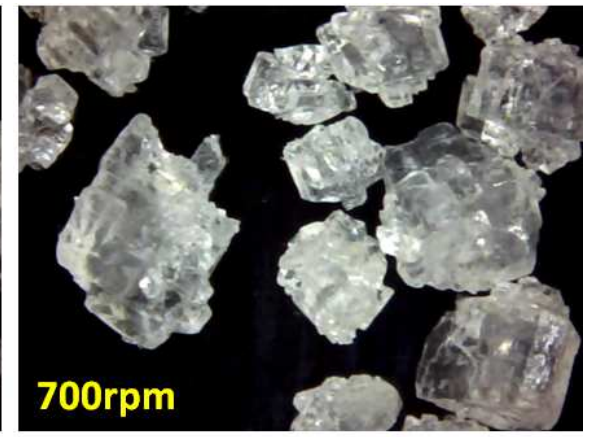

(b)

Figure 3. Crystal size of L-lysine in ST crystallizer with varied agitation speed.

The disadvantage of conventional ST crystallizer in terms of size distribution was originated from the non-homogeneous mixing condition. In general, the fluid velocity in ST crystallizer was widely dispersed, where it was only strong near the impeller, but it was quite weak as far from the impeller. For example, the fluid velocity was 0.7 times of impeller velocity near the impeller, while it was only $0.1-0.15$ times of impeller velocity as above or below the impeller [8]. As such, the hydrodynamic in ST crystallizer was a random of macro-, meso- and micro- fluid motion and contained a lot of stagnant fluid regions, which resulted in a nonhomogeneous mixing. Consequently, the supersaturation profile in ST crystallizer was nonhomogeneous, so the size distribution of crystal product was definitely non-uniformed. This problem of ST crystallizer became serious when the working volume was scaled up to industrial production. Thus, the more effective fluid hydrodynamic in other crystallizes has been exploited in recent years. For instance, Lawton et al [9] reported that the Oscillation baffles crystallizer having the vortices fluid motion in an inter-baffle zone provided a mono-dispersion of crystal product. Meanwhile, Oxley et al [10] indicated that the thin film fluid motion in the Spinning disk crystallizer having a high mass/heat transfer and homogeneous supersaturation profile facilitated the size distribution of crystal product. 


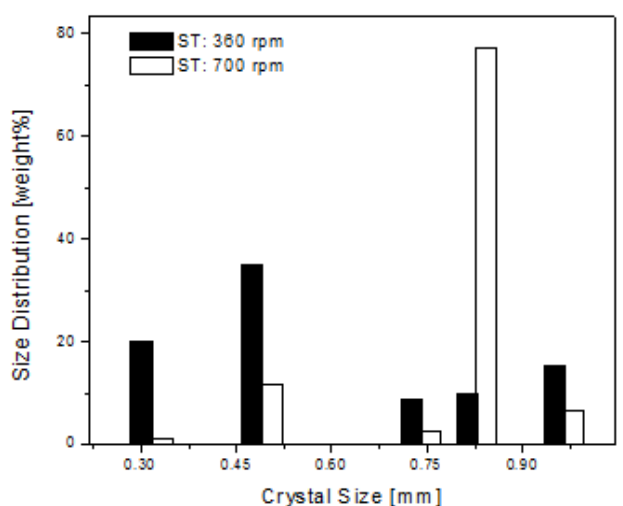

(a)

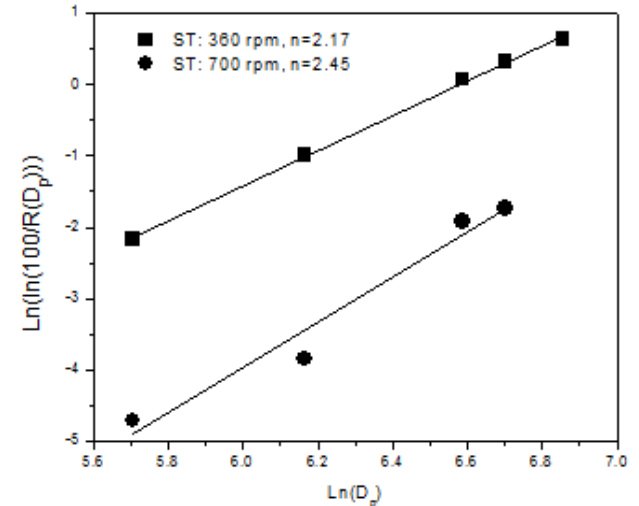

(b)

Figure 4. ST crystallizer with varied agitation speed: (a) size distribution, (b) coefficient size distribution (n).

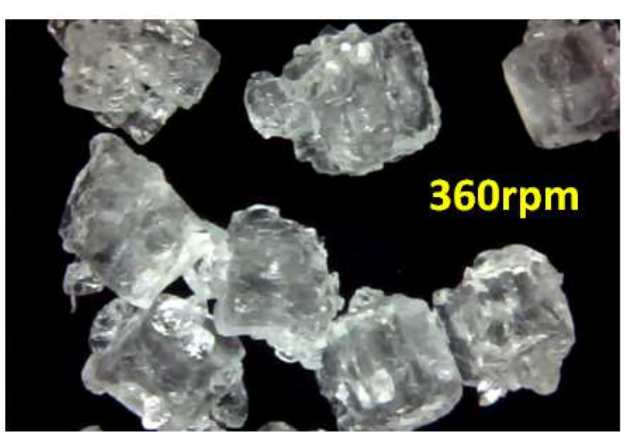

(a)

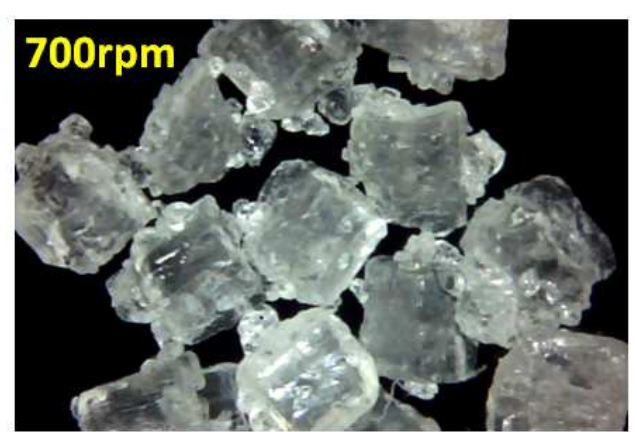

(b)

Figure 5. Crystal size of L-lysine in CT crystallizer with varied rotation speed.

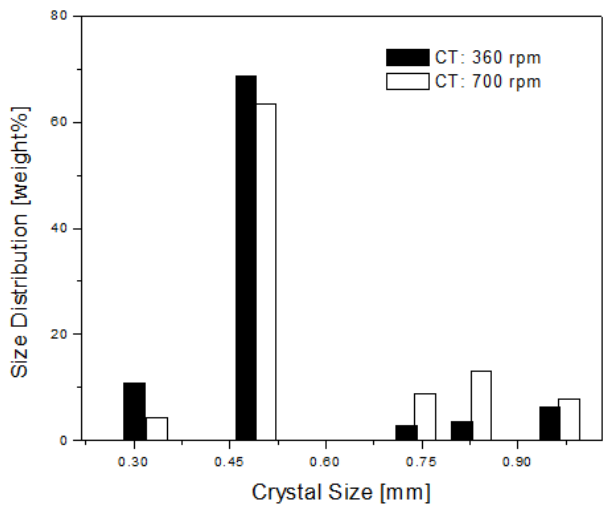

(a)

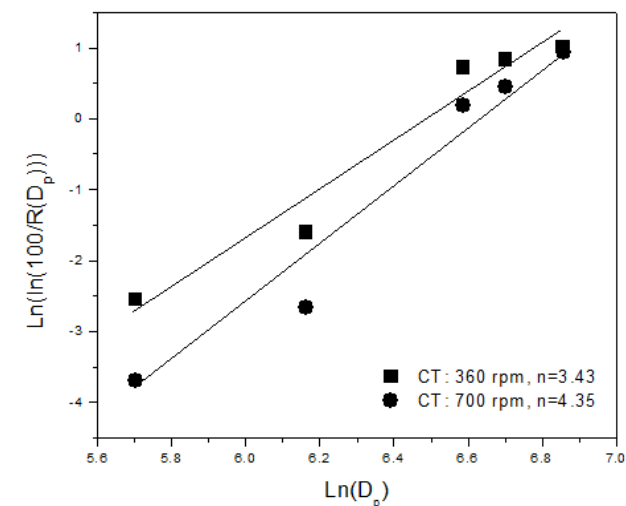

(b)

Figure 6. CT crystallizer with varied rotation speed: (a) size distribution, (b) coefficient size distribution (n).

In order to understand the advantage of CT crystallizer over the ST crystallizer with respect to the size distribution of L-lysine crystal product in cooling crystallization, the characteristic hydrodynamic of both crystallizers should be clearly understood. Here, the mixing intensity of 
fluid hydrodynamic in crystallizer is often represented via the energy dissipation per unit mass of suspension. In present study, the energy dissipation of the Taylor vortices flow in CT crystallizer was much higher than that of the random fluid motion in ST crystallizer at the same operating condition [4 - 5]. For example, when using the CT crystallizer, the energy dissipation was $0.45(\mathrm{~W} / \mathrm{kg})$ at $360 \mathrm{rpm}$ rotation speed of inner cylinder, while it was only $0.059(\mathrm{~W} / \mathrm{kg})$ in case of ST crystallizer at the same $360 \mathrm{rpm}$ agitation speed of impeller. As such, the inner cylinder of CT crystallizer provided 7.6 times higher energy to the suspension than that of the impeller in ST crystallizer [4 - 5]. That means the mixing condition of Taylor vortices flow in CT crystallizer was much more effective than that of the random fluid motion in ST crystallizer, implying that the supersaturation profile in CT crystallizer was much more homogeneous than that in ST crystallizer. As a result, the size distribution in CT crystallizer was certainly narrower than that in conventional ST crystallizer.

\section{CONCLUSIONS}

The present study explored the advantage of CT crystallizer over the conventional ST crystallizer with respect to promotion of size distribution of L-Lysine crystal product in the cooling crystallization. When using the CT crystallizer, the coefficient size distribution (n) was quite large as 3.43, while it was only 2.17 in case of ST crystallizer at the same $360 \mathrm{rpm}$, meaning that the size distribution of crystal product was significantly promoted as using the CT crystallizer compared to that of the ST crystallizer. The advantages of CT crystallizer over the ST crystallizer were explained in terms of the high energy dissipation of Taylor vortices flow, where it was 7.6 times higher than that of random fluid motion in ST crystallizer. As such, the supersaturation profile determined by the mixing condition in CT crystallizer was much more homogeneous than that of the ST crystallizer, which resulted in a narrow size distribution of Llysine crystal product.

Acknowledgment. This research was supported by the Vietnam Academy of Science and Technology (VAST).

\section{REFERENCES}

1. Myerson A. S. - Handbook of Industrial Crystallization, Oxford, 1993.

2. Alvarez A. J., and Myerson A. S. - Continuous Plug Flow Crystallization of Pharmaceutical Compounds, Cryst. Growth. Des. 10 (2010) 2219-2228.

3. Baldyga J., Podgorska W., and Pohorecki R. - Mixing-Precipitation Model with Application to Double Feed Semibatch Precipitation, Chem. Eng. Sci. 50 (1995) 12811300.

4. Tuan N. A., Joo Y. L., and Kim W. S. - Multiple Feeding Strategy for Phase Transformation of GMP in Continuous Couette - Taylor Crystallizer, Cryst. Growth. Des. 12 (2012) 2780-2788.

5. Tuan N. A., Kim J. M., Chang S. M., and Kim W. S. - Taylor Vortex Effect on Phase Transformation of Guanosine 5-Monophosphate in Drowning-out Crystallization, Ind. Eng. Chem. Res. 49 (2010) 4865-4872. 
6. Jung T., Kim W. S., and Choi C. K. - Effect of Nonstoichiometry on Reaction Crystallization of Calcium Carbonate in a Couette-Taylor Reactor, Cryst. Growth. Des. 4 (2004) 491-495.

7. Shan G., Igarashi K., Noda K., and Ooshima H. - Production of Large Crystals with a Narrow Crystal size Distribution by a Novel WWDJ Batch Crystallizer, Chem. Eng. J. 85 (2002) 161-167.

8. McCabe W. L., Smith J. C and Harriott P. - Unit Operations of Chemical Engineering, McGraw Hill, Boston (2001).

9. Lawton S., Steele G and Shering P. - Continuous Crystallization of Pharmaceuticals Using a Continuous Oscillatory Baffled Crystallizer, Org. Pro. Res. Dev. 13 (2009) 1357-1363.

10. Oxley P., Brechtelsbauer C., Ricard F., Lewis N and Ramshaw C. - Evaluation of Spinning Disk Reactor Technology for the Manufacture of Pharmaceuticals, Ind. Eng. Chem. Res. 39 (2000) 2175-2182. 